\title{
Roles of molecular chaperones in protein targeting to mitochondria
}

\author{
W. NEUPERT ${ }^{1}$ AND N. PFANNER ${ }^{2}$ \\ ${ }^{1}$ Institut für Physiologische Chemie der Universität München, Goethestrasse 33, 8000 München 2, F.R.G. \\ ${ }^{2}$ Institute für Biochemie der Universität Freiburg, Hermann-Herder-Str. 7, W-7800 Freiburg F.R.G.
}

\begin{abstract}
SUMMARY
Molecular chaperones are essential components of the machinery facilitating import of nuclear-encoded proteins into the mitochondria. They act at several steps of the complex import pathway. Cytosolic hsp 70 appears to contribute to maintaining precursors in a translocation-competent conformation. Mitochondrial hsp 70 has a distinct role in driving translocation across outer and inner mitochondrial membranes and probably in supporting unfolding of precursors in the cytosol. Hsp 60 in the matrix is involved in facilitating folding and assembly of imported polypeptide chains.
\end{abstract}

\section{INTRODUGTION}

During recent years molecular chaperones have proven to represent extremely versatile tools serving in a host of cellular processes. They were shown to exert essential functions in the replication of bacteriophages, to be involved in the assembly of supramolecular complexes, to be instrumental in facilitating folding of newly synthesized polypeptide chains, and to save proteins from the damaging effect of heat shock (Ellis 1987; Ellis \& Hemmingsen 1989; Rothman 1989). This is to list only a few of the many chaperone functions known at present. Most importantly, chaperones appear to exert these rather elaborate functions by a seemingly simple reaction, namely the reversible binding to non-native ('unfolded') structures on polypeptide chains. One example of how complex the functions of molecular chaperones can be is their central role in the reactions that direct newly synthesized polypeptide chains into the mitochondria.

We will first summarize our present knowledge on the molecular mechanisms of transport of nuclearencoded cytosolically synthesized proteins into the mitochondria. We will then describe how chaperones are involved in this process by going through the individual steps in which the various chaperones participate. In particular we will discuss: how chaperones in the cytosolic compartment are believed to assist in kecping precursor proteins in a transportcompetent state; how chaperones in the mitochondrial matrix are instrumental for driving the translocation of the polypeptide chains across the outer and inner mitochondrial membranes; how chaperones in the matrix facilitate the unfolding of precursor polypeptides in the cytosol in an indirect manner; how chaperones mediate the folding of newly imported chains in the mitochondrial matrix; and finally, how they participate in further sorting of some proteins to other mitochondrial subcomponents.

\section{EXPERIMENTAL FINDINGS}

\section{(a) Translocation of polypeptide chains from the cytosol into the mitochondrial matrix: \\ a multi-step process}

The many hundreds of different proteins which constitute the mitochondria are almost exclusively encoded by nuclear genes, synthesized on cytosolic ribosomes and released into the cytosol after completion of translocation (Horwich 1990; Pfanner \& Neupert 1990; Baker \& Schatz 1991). These proteins are made in the form of precursors most of which carry aminoterminal cleavable sequences which act as targeting signals. These signals have at least a twofold function: they enable the precursors to interact with receptors on the surface of the outer mitochondrial membrane; and, after crossing the outer membrane, they trigger the movement of the aminoterminus across the inner membrane. The transfer across outer and inner membranes is facilitated by separate machineries or 'translocation pores' in the two membranes (Hwang et al. 1991; Rassow \& Pfanner 1991). The translocation machineries cooperate closely in the transfer of precursors into the matrix. This cooperativity has been concluded from the accumulation of translocation intermediates spanning across both mitochondrial membranes. The sites at which translocation across both membranes takes place have been termed 'translocation contact sites' (Schleyer \& Neupert 1985). These translocation contact sites may be concentrated in areas where the two membranes are in close apposition, the so-called 'morphological contact sites'. The presence of an electrical membrane 
potential $\Delta \Psi$ across the inner membrane is an absolute requirement for starting translocation into or across the inner membrane. It is not entirely clear how $\Delta \Psi$ operates but a reasonable possibility is that the positively charged targeting sequences respond to $\Delta \Psi$ in an electrophoretic manner (Martin et al. 1991a). This would explain why $\Delta \Psi$ is only able (and required) to trigger the translocation of the aminoterminal part (i.e. the presequence) of the precursor, but does not play a role in the following translocation of the main part of the molecule.

After the precursor has partly or completely reached the matrix, the cleavable targeting sequence is removed by a soluble processing enzyme. Two components were found to be required for this reaction, the mitochondrial processing peptidase (MPP) and the processing enhancing protein (PEP) (Hawlitschek et al. 1988).

The proteins then undergo folding in the matrix to monomers and oligomers. In those cases where further sorting takes place, in particular to the inner membrane and the intermembrane space, reactions preventing folding must occur (see below). An important problem in this regard is in what conformational state precursors are crossing the two mitochondrial membranes. It has become clear already some time ago that unfolding of precursors is a prerequisite for transmembrane transfer (Eilers \& Schatz 1986). More recent studies have suggested that unfolding must be rather extensive. It is a likely possibility that polypeptides traversing the two membranes are in a fully extended state. Measurements of the length of the segment of a polypeptide chain that is spanning outer and inner membranes show that as little as 50-60 amino acid residues are sufficient to span the two membranes over a distance of about 15-20 nm (Rassow et al. 1990). This conclusion led to the suggestion that translocation occurs in a manner in which the polypeptide chain is sliding through a proteinaceous 'translocation pore' after more or less complete unfolding (Neupert et al. 1990).

\section{(b) A role of cytosolic hsp 70 s in preserving precursor proteins in a transport-competent state}

The conformational state of precursor proteins in the cytosol is usually different from that of the native functional protein. It had been noticed already quite early in the study of mitochondrial protein import that precursor proteins are extremely sensitive to added proteases under conditions where the functional counterparts are resistant. Furthermore, a consistent observation is the strong tendency of precursors towards aggregation, both with preproteins synthesized in cell-free systems and with preproteins expressed e.g. in Escherichia coli. In fact, precursor proteins made in $E$. coli can be dissolved in strongly denaturing media such as $8 \mathrm{M}$ urea, then diluted and imported into mitochondria; in most cases, however, such preparations are import-competent only for a short period of time, then aggregation takes place (summarized in Becker et al. 1992). These observations led to the conclusion that precursor proteins are in a conformational state that differs from that of their mature forms, being more unfolded and exposing hydrophobic 'sticky' patches.

It is therefore generally assumed that devices must exist in the cytosol to prevent misfolding and aggregation of preproteins. $\Lambda$ number of components have been implied in such a function. In particular, cytosolic hsp 70s, termed Ssal-4p in yeast, have been found to be important for the translocation of precursors into mitochondria (and also into the endoplasmic reticulum). In mutant strains in which three of the four SSA genes were deleted, precursors of certain mitochondrial proteins accumulated in the cytosol (Deshaies et al. 1988). Furthermore, experiments in vitro similarly suggested that cytosolic hsp 70 stimulates protein import into mitochondria (Murakami $e t$ al. 1988).

A few other cytosolic factors have also been described which bind to precursors in the cytosol but which do not belong to the family of heat shock proteins. Some of these factors have been implicated in interacting specifically with the targeting sequences (Murakami \& Mori 1990). It might well be that a diverse set of proteins acts on various parts of the precursor proteins in the cytosol using different strategies but having a common aim, namely the preservation of import-competence of precursors. In this respect mitochondrial import may resemble protein export from bacteria where hsp-type components, such as DnaK and GroEL, were found to interact with the presecretory proteins in the bacterial cytosol, but also more specialized chaperones such as SecB that do not belong to the group of heat shock proteins (Wickner et al. 1991; Phillips \& Silhavy 1990).

Protein import has a distinct requirement for $\mathrm{ATP}$ in the cytosol. This requirement is, at least in part, due to the action of the heat shock proteins. In particular, ATP hydrolysis seems to be required to release polypeptides from hsp 70s (Rothman 1989). This requirement can explain why hsp involvement and ATP hydrolysis are reactions that are usually found to parallel each other in experiments in vitro.

\section{(c) Hsp 70 in the mitochondrial matrix is essential for driving the translocation of precursors into the matrix space}

One of the eight hsp 70s of the yeast Saccharomyces cerevisiae is localized within the mitochondria (Craig et al. 1989). The product of this gene, called Ssclp or mitochondrial (mt-) hsp 70, performs essential functions in the cell as deletion of the $S S C I$ gene results in cell death (Craig et al. 1987). Mt-hsp 70 appears to be a general constituent of mitochondria since it was not only found in yeast, but also in humans, plants and protozoa.

$\Lambda$ temperature-sensitive mutant affected in SSC1 accumulated mitochondrial precursor forms (Kang et al. 1990). After a short time period following the shift from permissive to non-permissive temperatures, the preproteins were found with the mitochondrial fraction, yet they were not translocated into the mitochondria since they were accessible to protease added 
to the isolated mitochondria. Detailed biochemical analysis of the mutant phenotype revealed a dual role for this mt-hsp 70. It is involved in the translocation of precursors across the two mitochondrial membranes, and it plays an important role in the folding of precursors inside the mitochondria.

We will first discuss the experimental evidence for the role of $\mathrm{mt}$ hsp 70 in transmembrane movement of preproteins. When precursors were imported into mitochondria isolated from cells grown at a permissive temperature and then exposed to a non-permissive temperature, processing to the mature-sized proteins was observed to almost the same degree as in identically treated mitochondria from wild-type cells. However, when the mutant mitochondria were then probed as to whether they had imported these precursors into the matrix by treatment with protease, the answer was no. The mutant mitochondria accumulated the precursors in a two-membrane spanning fashion, i.e. the aminoterminus was able to enter the matrix space but the mature parts of the precursors were still exposed to the extramitochondrial space. These findings were made with matrix proteins as well as with proteins of the mitochondrial inner membrane and intermembrane space (Kang et al. 1990; Ostermann et al. 1990).

These data suggested that mitochondrial precursors have to interact with mt-hsp 70 to undergo transmembrane transfer, but that the onset for this requirement was only after the precursors had completed the initial translocation step that is triggered by the response of the targeting sequences to $\Delta \Psi$ across the inner membrane. Direct support for such a mechanism came from the demonstration that in the temperaturesensitive sscl-2 mutant (but not in the wild-type) complexes between the precursors and mt-hsp 70 could be isolated by immunoprecipitation with antibodies against mt-hsp 70. This suggests that in the mutant there may be a temperature-sensitive deficiency in the dissociation of mt-hsp 70 from the imported segments of the precursor polypeptides. Crosslinking experiments with a precursor protein arrested in a membrane spanning fashion showed that also in wild-type yeast precursors interact with mt-hsp 70 before translocation is completed (Scherer et al. 1990).

As discussed above, import of most precursor proteins not only requires $\mathrm{ATP}$ in the cytosol, but also $\Lambda T P$ in the matrix (Hwang \& Schatz 1989). Translocation across the inner membrane becomes arrested when the intramitochondrial ATP level is drastically reduced by incubating isolated mitochondria with apyrase (an enzyme which degrades ATP and ADP) and by inhibition of the mitochondrial ATP-synthase. $\Lambda \mathrm{TP}$ requirement in the matrix and the requirement for functional mt-hsp 70 were found to be closely correlated.

These findings are all compatible with a crucial role of mt-hsp 70 in driving the translocation of the precursors across the two mitochondrial membranes by binding to the unfolded segments appearing on the inside of the inner membrane (initially triggered by $\Delta \Psi)$, and thereby trapping the precursor chain step by step in the matrix space. ATP is required, according to such a mechanism, to confer a conformation competent for binding to mt-hsp 70, and $\Lambda \mathrm{TP}$ hydrolysis is required for the eventual release and recycling of mt-hsp 70 .

A salient feature of this proposed mechanism is the passage of the precursor into the matrix in an unfolded conformation. Only then would there be sufficient binding sites available for driving translocation in the direction of the matrix. We want to emphasize here that such a driving via hsp 70 binding may not necessarily be the only possible driving mechanism. In fact, we will discuss below that, with some kinds of precursors of intermembrane space proteins, the driving force may be provided by the export reaction from the matrix into the intermembrane space.

\section{(d) Hsp 70 in the matrix has a role in facilitating the unfolding of polypeptide chains on the mitochondrial surface}

If transmembrane movement requires an extensive unfolding of the polypeptide chain one may ask what the mechanisms are that facilitate unfolding on the cytosolic side. As discussed above, precursors are usually in a 'loosely folded' conformation but these conformations still contain abundant secondary and tertiary structure. Moreover, it has been found that in some cases domains of precursors assume a native conformation when they are in the cytosol. Various speculations have been made on the existence of unfolding components or 'unfoldases' that might interact with folded proteins in such a manner that the native folding state is disturbed. Sor far, no clear experimental evidence in favour of the existence of such unfoldases has been obtained.

A mechanism for unfolding can be proposed, however, relying on further data obtained through the analysis of the temperature-sensitive ssc1-2 mutant. The step of ATP-dependent unfolding on the mitochondrial surface can be bypassed by artificially unfolding precursors before import. In particular, this was done with fusion proteins consisting of aminoterminal segments of the ATPase subunit 9 precursor and of complete mouse cytosolic dihydrofolate reductase (DHFR). Upon unfolding (in $8 \mathrm{~m}$ urea followed by rapid dilution), these Su9-DHFR precursors can be imported into isolated mitochondria without addition of ATP, in contrast to import of the same precursors out of reticulocyte lysate where they presumably are complexed with hsp 70 .

Import of these unfolded fusion proteins into isolated mitochondria from the ssc1-2 mutant was found to occur with almost the same kinetics as import into wild-type mitochondria, in sharp contrast to the situation with import from the reticulocyte lysate system (Kang et al. 1990). This observation demonstrated that the mutant mitochondria mainly had problems with importing precursors into the matrix when unfolding was a critical factor. Unfolding on the mitochondrial surface, i.e. of those parts of a precursor that are still present in the cytosolic compartment, is 
apparently facilitated by mitochondrial hsp 70 together with matrix ATP.

How could a component in the matrix then facilitate the unfolding of a protein on the other side of two membranes? $\Lambda$ s an answer to this question we propose that unfolding in the cytosol is essentially a spontaneous process. We suggest that unfolding occurs in very small steps that require a very low amount of energy. Each time such a small segment has assumed an extended conformation it could slide in the 'translocation pore' and mt-hsp 70 on the other side would have the opportunity to bind and make the process eventually irreversible. Thereby, the equilibrium process of folding and unfolding could be turned into a vectorial reaction.

Experimental support for this pathway of unfolding comes from the following observations. Membrane spanning intermediates of cyt $b_{2}$-DHFR fusion proteins (aminoterminal parts of the cytochrome $b_{2}$ precursor fused to complete DHFR) can be accumulated in the presence of methotrexate which stabilizes the DHFR domain. Import occurs until the folded DHFR domain 'hits' the outer membrane (Rassow et al. 1989). Stabilization by the ligand clearly reduces spontaneous unfolding strongly. The free energy of stabilization is still rather low, being in the range of a few kcal per mole. This is a very small amount of energy compared with that needed for the $\Lambda \mathrm{TP}$ dependent release of the bound mt-hsps. Although it is not clear how many molecules of matrix ATP are required for importing an average-sized mitochondrial matrix protein, the free energy needed for preventing unfolding (e.g. by stabilization through a ligand) is almost certainly negligible compared to the free energy of ATP hydrolysis needed for recycling the mthsps. Also in agreement with the proposed unfolding mechanism is the fact that the translocation of the folded DHFR domain (when methotrexate is removed after accumulating transmembrane cyt $b_{2}$-DHFR) is much more dependent on temperature as compared to the import of the urea-unfolded precursor (Eilers et al. 1988; Rassow et al. 1989). In summary, mt-hsp 70 in an indirect manner appears to play a decisive role in the unfolding of precursor proteins in the cytosol.

\section{(e) Hsp 70 and hsp 60 in the matrix are involved in folding of polypeptide chains}

Mitochondria from all sources analysed so far contain the chaperonin hsp 60 which is structurally and functionally closely related to GroEL of prokaryotic organisms (Reading et al. 1989; Ellis \& Hemmingsen 1989). This similarity appears to reflect the endosymbiotic origin of mitochondria although hsp 60 of mitochondria is encoded by a nuclear gene. Like mt-hsp 70, hsp 60 is essential for the life of yeast cells (Cheng et al. 1989).

The analysis of the yeast mutant mift, which is deficient in hsp 60 function in a temperature-sensitive manner, revealed an unimpeded import of precursors into the matrix, but a lack of formation of active matrix enzymes such as ornithine transcarbamoylase, $F_{1}$-ATPase or hsp 60 itself (Cheng et al. 1989). This result was found both in vivo and after import of proteins into isolated mitochondria. Further biochemical investigation then showed that hsp 60 is directly involved in the folding of individual polypeptide chains. For its activity in facilitating folding, hsp 60 requires $\mathrm{ATP}$ hydrolysis. Upon reduction of the $\Lambda T P$ level in the matrix, in the presence of a nonhydrolysable $\triangle T P$ analogue, $\Lambda \mathrm{MP}-\mathrm{PNP}$, or at reduced temperature, a slow-down of folding in the matrix was observed and the imported precursors were largely found in association with the hsp 60 complex (Ostermann et al. 1989). These results gave the first experimental indication that folding of individual polypeptide chains in vivo is mediated by a chaperone.

However, not only hsp 60 is involved in facilitating folding in the matrix. With the ssc1-2 mutant it was found that the refolding of urea-unfolded precursors imported at non-permissive temperature was strongly reduced. The precursors remained associated with mthsp 70 and were presumably not transferred to hsp 60 (Kang el al. 1990). It was therefore suggested that precursors have to be passed on from mt-hsp 70 to hsp 60 to become folded (Neupert et al. 1990; Langer et al. 1992). This view has been questioned, however, and it has been claimed that pools of precursors not complexed with either hsp 70 or hsp 60 exist (ManningKrieg et al. 1991).

\section{(f) Hsp 70 and hsp 60 play important roles in intramitochondrial sorting to the inner membrane and the intermembrane space}

Some proteins that are transported to the matrix do not remain there but are sorted to other submitochondrial compartments. Several lines of evidence suggest that molecular chaperones in the matrix are involved in these processes. As an example, the precursor of the Rieske Fe-S protein (a component of complex III of the respiratory chain anchored to the inner membrane and exposing a hydrophilic domain into the intermembrane space) requires both mt-hsp 70 and hsp 60 for becoming localized to its functional site. In the sscl-2 mutant as well as in the mif 4 mutant its assembly was found to be impaired (Kang et al. 1990; Cheng et al. 1989). A similar situation has been described for cytochrome $b_{2}$ (a soluble component of the intermembrane space) (Koll et al. 1992), although this pathway has recently been challenged (Glick et al. 1992).

What is the role of the mitochondrial chaperones in these cases? The role of mt-hsp 70 appears to be to facilitate the entry of the polypeptides into the matrix in the same way as it facilitates entry of matrix components. $A$ function of hsp 60 was proposed to lie in the protection of the intermediates in the matrix against misfolding or aggregation before the ensuing export reaction (Koll et al. 1992). The absence of folding by hsp 60 in these cases was thought to be the result of the presence of sequences in the intermediates, the so-called sorting sequences, which would prevent acquisition of a native folding state. It should be pointed out that this mode of sorting is likely to be the preferred one when import into the matrix is very 

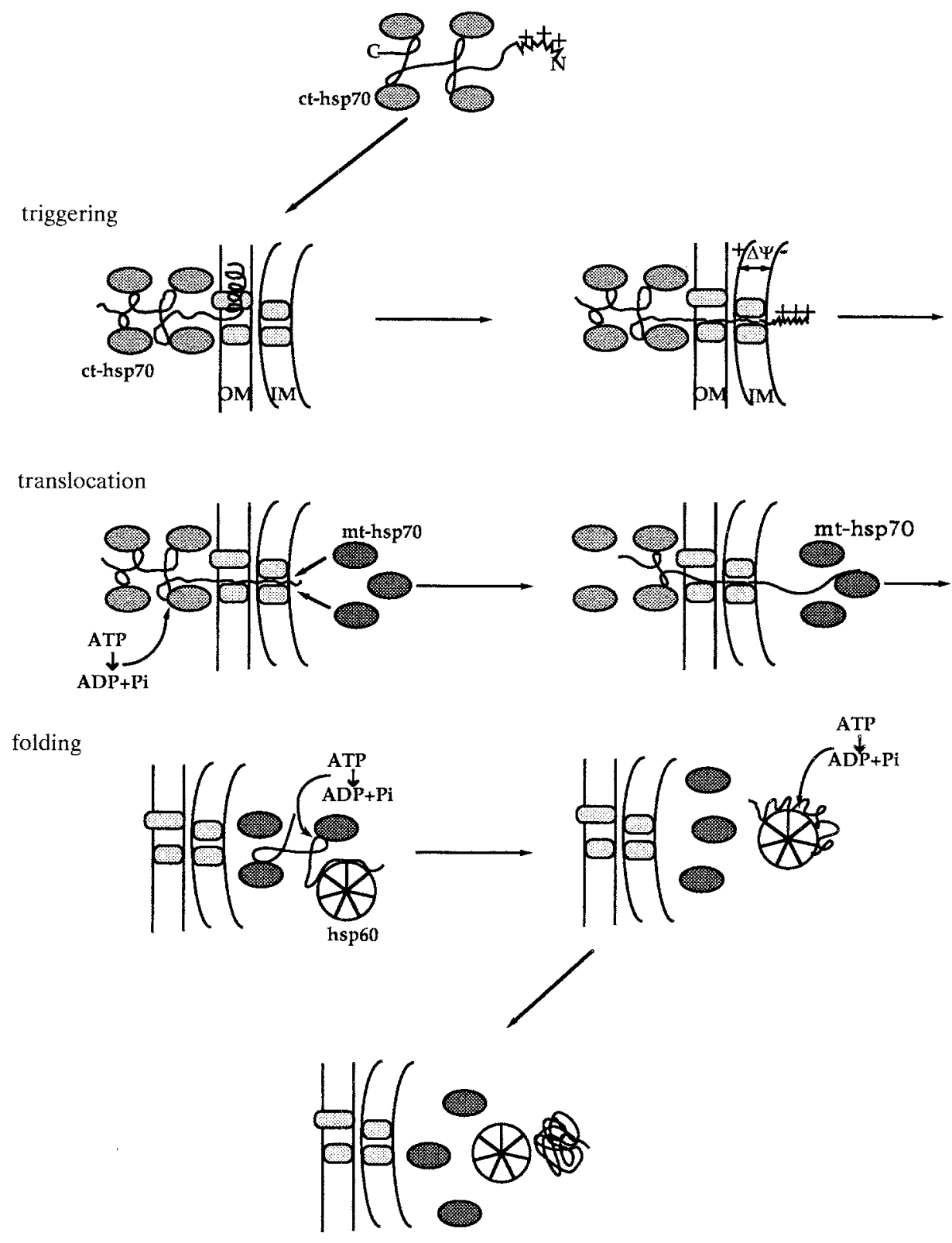

Figure 1. Hypothetical model on the roles of molecular chaperones in mitochondrial protein import (modified after Neupert et al. 1990). C, carboxylterminus of preprotein; IM, inner membrane; N, aminoterminus of preprotein; OM, outer membrane.

rapid. In the experiments described, in fact, import was performed using urea-unfolded precursors whose import is 10-100-fold faster than import out of reticulocyte lysate. In the former case, export may not keep up in pace with import, and therefore hsp 70 and hsp 60 come into play.

It appears that in some cases both mt-hsp 70 and hsp 60 can be bypassed, in particular when export is faster than import. With certain precursor proteins, in particular with cyt $b_{2}$-DHFR fusion proteins in which the pre-cyt $b_{2}$ part is shorter than approximately 170 amino acid residues, a participation of $\mathrm{mt}$-hsp 70 and hsp 60 was not observed. In agreement with this, there was also no requirement for matrix $\Lambda T P$ (unpublished results). It appears likely that under such conditions import into the matrix and export into the intermembrane space are coupled events. The driving force for the overall reaction might then be provided by the transport into the intermembrane space, rather than by mt-hsp 70 binding. This specialized pathway is, however, apparently not possible when longer segments of pre-cyt $b_{2}$ precede the DHFR domain, possibly because the energy of the export reaction is not sufficient to drive import.

\section{CONGLUSIONS AND PERSPEGTIVES}

Our present knowledge and ideas about the roles of molecular chaperones in mitochondrial protein sorting are summarized in a working hypothesis which is depicted in figure 1 . The overall reaction is divided into three consecutive steps.

1. Triggering step. The hsp 70-complexed preproteins 
interact with the receptor machinery in the outer membrane and translocation of the aminoterminal targeting signal into the mitochondrial matrix takes place.

2. Translocation step. Spontaneous unfolding on the cytosolic side, sliding of the extended precursor chain in a 'translocation pore', and binding of mt-hsp 70 on the matrix side lead to gradual movement of the precursor into the matrix. ATP is required on both sides of the two mitochondrial membranes.

3. Folding step. The precursors are passed on from mt-hsp 70 to hsp 60 and become folded on the chaperonin. ATP is required for release of $\mathrm{mt}$-hsp 70 and for repeated cycles of binding and release of the polypeptide on hsp 60 in the course of the folding process.

It may be useful to look at the functions of mitochondrial molecular chaperones from an evolutionary angle. The prokaryotic origin of the mitochondria is reflected in the amazing similarities of the molecular roles of hsp 60 and groEL (Ellis \& Hemmingsen 1989; Martin et al. 1991b). Mitochondrial hsp 70 is a very close relative of bacterial DnaK (Craig et al. 1989). Why should the mitochondrial equivalent of DnaK have acquired such a specific role in driving the import of proteins which in the prokaryotic ancestors of the mitochondria were encoded by the DNA of the endosymbiont? The main function of DnaK/hsp 70 chaperones appears to be binding to unfolded segments of polypeptide chains in a reversible fashion (Flynn et al 1989). One can easily imagine that such a rather simple and general tool can be used for controlling a large host of cellular reactions. Mitochondrial import may have evolved taking advantage of two basic devices that could have existed already in the prokaryotic ancestors. This first device would be a controllable 'pore' that allows the passage of polypeptides through the envelope in an extended state; the existence of pores, for example for the uptake of polypeptides such as colicin A (Bourdineaud et al. 1989), might indicate that such an assumption is not entirely unreasonable. The DnaK in the prokaryotic ancestor would then be a complementary tool as it was especially designed to bind to extended parts of polypeptide chains. These two devices together would then have served to set up the basic machinery for protein import into endosymbiotic organelles.

It should be made clear at this point that the working hypothesis illustrated in figure 1 , if true in all parts, is certainly far from being complete. It is very likely that additional protein components are involved in the complex reaction scheme. From bacteria we know that, for instance, in addition to DnaK the heat shock proteins DnaJ and GrpE exert important functions in chaperone action (Georgopoulos et al. 1990; Langer et al. 1992). It is not unlikely that related components also function in mitochondria. In yeast, a DnaJ homologue has already been described which could have such a role, although a mitochondrial location in this case has not been proven (Blumberg \& Silver 1991). The proposed reaction scheme is also not meant to indicate that all precursor proteins must use the same pathway and the same components in an identical manner. Alternative pathways, using unknown components or bypass pathways, should always be considered as being possible. This may be particularly true because the folding behaviour and the binding capacity for molecular chaperones may not be uniform properties of all precursor proteins.

Future investigations will have to aim at a critical examination of our present working hypothesis. They will have to detect a presumably large number of additional chaperones and related components, they must define the molecular mechanisms of chaperone action in mitochondria to provide a much more precise picture, and they will have to search for additional functions of the known mitochondrial chaperones.

We are grateful to Alcxandra Wcinzicrl for preparing the drawing. Studies from the authors' laboratories werc supported by the Deutsche Forschungsgemeinschaft, the Human Frontier Science Program and the Fonds der Chemischen Industric.

\section{REFERENGES}

Bakcr, K.P. \& Schatz, G. 1991 Mitochondrial protcins cssential for viability mediate protcin import into ycast mitochondria. Nature, Lond. 349, 205-208.

Becker, K., Guiard, B., Rassow, J., Söllner, T. \& Pfanner, N. 1992 Targeting of a chemically pure preprotein to mitochondria does not require the addition of a cytosolic signal rccognition factor. J. biol. Chem. 267, 5637-5643.

Blumberg, H. \& Silver, P.A. 1991 A homologuc of the bacterial heat-shock gene DnaJ that alters protein sorting in ycast. Nature, Lond. 349, 627-630.

Bourdincaud, J.-P., Howard, S.P. \& Lazdunski, C. 1989 Localization and asscmbly into the Escherichia coli cnvelope of a protcin required for entry of colicin A. J. Bact. 171, 2458-2465.

Cheng, M.Y., Hartl, F.-U., Martin, J., Pollock, R.A., Kalousck, F., Ncupert, W., Hallberg, E.M., Hallberg, R.L. \& Horwich, A.L. 1989 Mitochondrial heat-shock protcin hsp 60 is esscntial for assembly of protcins imported into yeast mitochondria. Nature, Lond. 337,620 625

Craig, E.A., Kramer, J. \& Kosic-Smithers, J. 1987 SSC1, a member of the 70-kDa heat shock protein multigene family of Saccharomyces cerevisiae, is csscntial for growth. Proc. nain. Acad. Sci. U.S.A. 84, 4156-4160.

Craig, E.A., Kramer, J., Shilling, J., Werner-Washburne, M., Holmes, S., Kosic-Smithers, J. \& Nicolet, C.M. 1989 SSC1, an cssential member of the ycast HSP 70 multigcne family, encodes a mitochondrial protcin. Molec. Cell. Biol. 9, 3000-3008.

Deshaics, R.J., Koch, B.D., Werner-Washburne, M., Craig, E.A. \& Sheckman, R. 1988 A subfamily of stress proteins facilitates translocation of secretory and mitochondrial precursor polypcptides. Nature, Lond. 332, 800-805.

Eilcrs, M. \& Schatz, G. 1986 Binding of a specific ligand inhibits import of a purified precursor protein into mitochondria. Nature, Lond. 322, 228232.

Eilers, M., Hwang, S. \& Schatz, G. 1988 Unfolding and refolding of a purified precursor protein during import into isolated mitochondria. EMBO J. 7, 1139-1145.

Ellis, J. 1987 Proteins as molccular chaperones. Nalure, Lond. 328, 378-379.

Ellis, R.J. \& Hemmingsen, S.M. 1989 Molecular chaper- 
ones: proteins essential for the biogenesis of some macromolecular structures. Trends Biochem. Sci. 14, 339-342.

Flynn, G.C., Chappell, 'T.G. \& Rothman, J.E. 1989 Peptide binding and release by proteins implicated as catalysts of protein assembly. Science, Wash. 245, 385-390.

Georgopoulos, C., Ang, D., Liberek, K. \& Zylicz, M. 1990 Properties of the Escherichia coli heat shock proteins and their role in bacteriophage $\lambda$ growth. In Stress proteins in biology and medicine (ed. T. Morimoto, A. Tissiers \& C. Georgopoulos), pp. 191-221. New York: Cold Spring Harbor Laboratory Press.

Glick, B.S., Brandt, A., Cunningham, K., Müller, S., Hallberg, R.L. \& Schatz, G. 1992 Cytochromes $c_{1}$ and $b_{2}$ are sorted to the intermembrane space of yeast mitochondria by a stop-transfer mechanism. Cell 69, 809-822.

Hawlitschek, G., Schneider, H., Schmidt, B., Tropschug, M., Hartl, F.-U. \& Neupert, W. 1988 Mitochondrial protein import: identification of processing peptidase and of PEP, a processing enhancing protein. Cell 53, 795-806.

Horwich, A. 1990 Protein import into mitochondria and peroxisomes. Curr. Opin. Cell Biol. 2, 625-633.

Hwang, S.T. \& Schatz, G. 1989 Translocation of proteins across the mitochondrial inner membrane, but not into the outer membrane, requires nucleoside triphosphates in the matrix. Proc. natn. Acad. Sci. U.S.A. 86, 8432-8436.

Hwang, S.I., Wachter, C. \& Schatz, G. 1991 Protein import into the yeast mitochondrial matrix: a new translocation intermediate between the two mitochondrial membranes. J. biol. Chem. 266, 21083-21089.

Kang, P.J., Ostermann, J., Schilling, J., Neupert, W., Craig, E.A. \& Pfanner, N. 1990 Requirement for hsp 70 in the mitochondrial matrix for translocation and folding of precursor proteins. Nature, Lond. 348, 137-143.

Koll, H., Guiard, B., Rassow, J., Ostermann, J., Horwich, A.L., Neupert, W. \& Hartl, F.-U. 1992 Antifolding activity of hsp 60 couples protein import into the mitochondrial matrix with export to the intermembrane space. Cell 68, 1163-1175.

Langer, 'I., Lu, C., Echols, H., Flanagan, J., Hayer, M.K. \& Hartl, F.-U. 1992 Successive action of DnaK, DnaJ and GroEL along the pathway of chaperone-mediated protein folding. Nature, Lond. 356, 683-689.

Manning-Krieg, U.C., Scherer, P.E. \& Schatz, G. 1991 Sequential action of mitochondrial chaperones in protein import into the matrix. EMBO J. 10, 3273-3280.

Martin, J., Mahlke, K. \& Pfanner, N. 1991 a Role of an energized inner membrane in mitochondrial protein import: $\Delta \Psi$ drives the movement of presequences. J. biol. Chem. 266, 1805I-18057.

Martin, J., Langer, T., Boteva, R., Schramel, A., Horwich, A.L. \& Hartl, F.-U. $1991 b$ Chaperonin-mediated protein folding at the surface of groEL through a 'molten globule'-like intermediate. Nature, Lond. 352, 36-42.

Murakami, H., Pain, D. \& Blobel, G. 1988 70-kD heat shock-related protein is one of at least two distinct cytosolic factors stimulating protein import into mitochondria. J. Cell Biol. 107, 2051-2057.

Murakami, K. \& Mori, M. 1990 Purified presequence binding factor (PBF) forms an import-competent complex with a purified mitochondrial precursor protein. EMBO J. 9, 3201-3208.

Neupert, W., Hartl, F.-U., Craig, E.A. \& Pfanner, N. 1990 How do polypeptides cross the mitochondrial membranes? Cell 63, 447-450.

Ostermann, J., Horwich, A.L., Neupert, W. \& Hartl, F.-U. 1989 Protein folding in mitochondria requires complex formation with hsp 60 and A'P hydrolysis. Nature, Lond. 341, 125-130.

Ostermann, J., Voos, W., Kang, P.J., Craig, E.A., Neupert,
W. \& Pfanner, N. 1990 Precursor proteins in transit through mitochondrial contact sites interact with hsp 70 in the matrix. FEBS Lett. 277, 281-284.

Pfanner, N. \& Neupert, W. 1990 The mitochondrial protein import apparatus. A. Rev. Biochem. 59, 331-353.

Phillips, G.J. \& Silhavy, T.J. 1990 Heat-shock proteins DnaK and GroEL facilitate export of LacZ hybrid proteins in E. coli. Nature, Lond. 344, 882-884.

Rassow, J. \& Pfanner, N. 1991 Mitochondrial preproteins en route from the outer membrane to the inner membrane are exposed to the intermembrane space. FEBS Lett. 293, 85-88.

Rassow, J., Guiard, B., Wienhues, U., Herzog, V., Hartl, F.-U. \& Neupert, W. 1989 'Translocation arrest by reversible folding of a precursor protein imported into mitochondria. A means to quantitate translocation contact sites. J. Cell Biol. 109, 1421-1428.

Rassow, J., Hartl, F.-U., Guiard, B., Pfanner, N. \& Neupert, W. 1990 Polypeptides traverse the mitochondrial envelope in an extended state. FEBS Lett. 275, 190194.

Reading, D.S., Hallberg, R.L. \& Myers, A.M. 1989 Characterization of the yeast HSP 60 gene coding for a mitochondrial assembly factor. Nature, Lond. 337, 655659.

Rothman, J.E. 1989 Polypeptide chain binding proteins: catalysts of protein folding and related processes in cells. Cell 59, 591-601.

Scherer, P.E., Krieg, U.C., Hwang, S.T., Vestweber, D. \& Schatz, G. 1990 A precursor protein partly translocated in to yeast mitochondria is bound to a $70 \mathrm{kd}$ mitochondrial stress protein. EMBO J. 9, 4315-4322.

Schleyer, M. \& Neupert, W. 1985 Transport of proteins into mitochondria: translocational intermediates spanning contact sites between outer and inner membranes. Cell 43 , 339-350.

Wickner, W., Driessen, A.J.M. \& Hartl, F.-U. 1991 'The enzymology of protein translocation across the Escherichia coli plasma membrane. A. Rev. Biochem. 60, 101-124.

\section{Discussion}

W. J. Welch (Department of Medicine and Physiology, University of California, San Franscisco, U.S.A.). Why do proteins destined for the intermembrane mitochondrial space have to interact with hsp 60? If hsp 60 assists protein folding does this mean that the ironsulphur protein is active in the matrix?

W. Neupert. I imagine that hsp 60 interacts with most proteins that enter the matrix space and tries to assist its folding: it certainly does this even with foreign proteins like DHFR. But the protein may not be capable of being folded because a signal sequence is still present, or because of the presence of other factors which prevent folding. So the extent of interaction with hsp 60 will depend on these factors, and on the kinetic situation, and may not be obligatory for proteins destined for the intermembrane space. The situation could resemble that suggested for the role of GroEL in protein transport in bacteria.

W. J. WeLch. Do the authors think there may be a role for TCPI in binding to mitochondrial precursor proteins in the cytosol? 
W. Neupert. I have no evidence on which to assess this possibility.

A. Baker (Department of Biochemistry, University of Cambridge, U.K.). The authors suggest that the two different models for protein transport into the intermembrane space can be reconciled by taking into account kinetic factors. It is possible to change the pathway of transport of, for example, cytochrome $b_{2}$, by altering the rate of transport?

W. Neupert. Kinetic factors will not be able to reconcile the two models, as they are mechanistically different, however, they may explain controversial interpretations of experimental data. Since the two models are not mutually exclusive it cannot be excluded that different pathways might be favoured by different experimental conditions.

A. BAKER. In the experiments with protein import into mitochondria from the hsp 60 mutant where the authors found no mature form of the iron-sulphur protein, did they observe any mature protease-protected form of cytochrome $b_{2}$ ?

W. Neupert. There could be some present, but clearly less than in the control mitochondria.

M.-J. Gething (Howard Hughes Medical Institute, University of Texas, Dallas, U.S.A.). If, as the authors suggest, the driving force for protein import into the matrix space is binding to the matrix hsp 70, what is the driving force for the transport from the matrix to the intermembrane space? This situation seems to parallel the transport of proteins into the periplasmic space of bacteria.

W. Neupert. We do not know the answer to this interesting question. $\Lambda$ t the moment we can study only the import and export steps at the same time, and what we require for such studies is a vesicle system which will transport from the matrix. There is of course $\Lambda \mathrm{TP}$ in the intermembrane space, but how proteins fold in this space is an open question.

R. Jaenicke (Department of Biophysics and Physical Biochemistry, University of Regensburg, F.R.G.). How long does the precursor protein on the cytosolic side of the mitochondrial membrane have to be to interact with hsp 70?

W. Neupert. There is no systematic study of this aspect. Cytochrome $c$ is a small mitochondrial protein and we have been unable to see any interaction between this protein and hsp 70; the transport of this protein across the outer membrane in to the intermembrane space does not require $\Lambda \mathrm{TP}$ on the cytosolic side. 